\title{
Searching for Rational Bubble Footprints in the Singaporean and Indonesian Stock Markets
}

\begin{abstract}
We re-examine the presence of rational speculative bubbles in the Singaporean and Indonesian stock markets in light of contradictory results in the literature. We employ a mix of descriptive statistics, explosiveness tests and duration dependence tests for an expanded dataset from 1970 to 2013 that covers at least two suspected bubble episodes - the 1997 Asian Financial Crisis (AFC) and the Global Financial Crisis (GFC). We find bubble footprints in Singapore and Indonesia using descriptive statistics and explosiveness tests. However, we find no evidence of rational bubbles in Singapore using the duration dependence test. On the other hand, in Indonesia we find evidence of rational bubbles in weekly but not in monthly data. Our results indicate that the duration dependence test results could be sensitive to data frequency suggesting that the duration dependence test results are not always conclusive and that it should be used in conjunction with other tests.
\end{abstract}

JEL Classification: C41, G12

Keywords: duration dependence; rational speculative bubbles; Singapore; Indonesia 


\section{Searching for Rational Bubble Footprints in the Singaporean and Indonesian Stock Markets}

\section{Introduction}

Since the 1970s, both the Singaporean and Indonesian stock markets witnessed several periods of price inflation, followed by sharp drops. Many consider these cycles of boom and busts as evidence of asset bubbles. Bubbles are characterized by an extended inflation in stock prices or returns that attain a peak, and then suddenly crash. The popular press usually attributes bubbles to irrational investors' behaviour as herding, fashions, or fads drive their expectations and their irrational optimism (Schiller, 2000). However, some bubbles could develop entirely from rational behaviour of investors. Rational speculative bubbles could continue growing although investors know a bubble had formed as long as the probability of a continued gain exceeds the probability of a loss. ${ }^{1}$

McQueen and Thorley (1994) showed that rational bubbles possess negative duration dependence. This means that the conditional probability of a positive run of returns ending, falls with the length of the run. As a rational bubble inflates, unforeseen price changes originate from unanticipated changes in the fundamental value of the stock and unexpected changes in the bubble. As a bubble continues growing, it begins dominating the fundamental component. Hence, a negative shock to the fundamental component would have little impact on total returns. Thus, the bubble continues growing despite these shocks, and the larger the bubble inflates, the less effect a negative shock would have on total returns, hence yielding the negative duration dependence. The bubble continues growing until a significant negative innovation bursts the bubble.

We search for rational bubbles in Indonesia and Singapore because Indonesia is the largest economy in the ten-nation ASEAN block, while Singapore is ASEAN's financial centre. ${ }^{2}$ Singapore has traditionally enjoyed the biggest share of foreign direct investments (FDIs) in the ASEAN, followed by Indonesia. The presence of bubbles in these economies has important implications on market efficiency, allocation of resources, and asset pricing. A collapsing bubble inflicts damage on an economy as it can trigger capital flight, a depreciating currency, and rising unemployment. Thus, bursting bubbles could lead to instability in the economy and spread to other countries via contagion. Prior research has tested for the presence of bubbles in Singapore

\footnotetext{
${ }^{1}$ McQueen and Thorley (1994, p.379) define a rational bubble as one where "the probability of a high return exactly compensates investors for the probability of a crash".

2 ASEAN stands for Association of Southeast Asian Nations. The other member states of the ASEAN are Thailand, Malaysia, Philippines, Vietnam, Myanmar, Brunei, Cambodia, and Laos.
} 
and Indonesia but the results are mixed and contradictory. Sarno and Taylor (1999) use cointegration tests and report the presence of stock market bubbles in both Singapore and Indonesia over the period from 1988 to 1997. In a more recent study, Ahmed, Rosser and Uppal (2010) employ regime-switching tests and also report the presence of non-linear speculative bubbles in both Singapore and Indonesia over the period from 1990 to 2006. In contrast, using duration dependence tests, Rangel and Pillay (2007) report the absence of rational bubbles in Singapore over the period from 1975 to 2007, while Yu and Hassan (2009) also using duration dependence tests report the absence of bubbles in Indonesia over the period from 1992 to $2003 .^{3}$ Though the sample periods for the duration dependence tests of Rangel and Pillay (2007) and Yu and Hassan (2009) encompass the 1997 Asian Financial Crisis (AFC), they both miss the Global Financial Crisis (GFC) of 2007-2008. This is significant since if their sample period contained only one bubble episode this could severely affect the power of their duration dependence tests (Chan, McQueen and Thorley, 1998).

In this study, we employ a mix of descriptive statistics, explosiveness tests and duration dependence tests to search for evidence of rational speculative bubbles in the Singaporean and Indonesian stock markets between 1970 and 2013. Hence our data set includes at least two alleged bubble episodes - the AFC and the GFC, unlike in Rangel and Pillay (2007) and Yu and Hassan (2009) whose sample period only encompass the AFC. Consequently, our duration dependence tests should have more power than theirs. In addition we use an extended sample period that is 120 (520) months (weeks) longer than Yu and Hassan’s (2009) and 75 (331) months (weeks) longer than Rangel and Pillay’s (2007) which also adds to the power of our tests. The two alleged bubbles and longer sample period would strengthen the confidence of our findings and help resolve the conflicting results of previous studies.

The duration dependence test for rational bubbles is superior to the other tests since negative duration dependence is unique to rational bubbles (McQueen and Thorley, 1994). However Harman and Zuehlke (2004) and Lehkonen (2010) have recently questioned the efficacy of the duration dependence test by suggesting that the test results are sensitive to data frequency. We also test for the practical efficacy of the duration dependence

\footnotetext{
${ }^{3}$ Rangel and Pillay's (2007) sample end in January 2007. Though they report significant duration dependence in runs of negative returns, Chan et al. (1998) attributes such duration dependence to chance or other causes such as fads and not due to rational bubbles since rational bubbles cannot be negative.
} 
test in our study by using both weekly and monthly returns in our tests. ${ }^{4}$ Both Rangel and Pillay (2007) and Yu and Hassan (2009) conducted their duration dependence tests only on monthly returns.

There is currently no cogent theory of how bubbles develop and then burst (Chan et al., 1998). Innocuous events or a major incident such as a terrorist attack could trigger steep declines in equity markets (Ramiah and Graham, 2013). Furthermore, institutional investors could spark a selloff by systematically using signals from insider trading as they decipher poor earnings reports or problems before the public finds out (Wang, 2011). Finally, managers with their earnings tied to stock prices may inflate their companies' earnings that boost stock prices higher while they sell and profit from greater insider trading (Huddart and Louis, 2007). Once the correction starts, the bubble burst and stock prices begin plummeting. ${ }^{5}$

Researchers developed four categories of bubble tests in the literature. For the first category, researchers examine the descriptive statistics of return distributions to determine the presence of a bubble. As a bubble develops, the return distributions show autocorrelation, skewness and kurtosis. The long run up in prices characteristic of bubbles means returns are positively autocorrelated. The unusually large negative returns that occurs when the bubble bursts also means that the return distribution is negatively skewed and leptokurtic (i.e., have fat tails). McQueen and Thorley (1994) also indicate that as the bubble grows, the mixing of low variance distributions associated with small bubbles with high variance distributions as the bubble grows results in fat tails in return distributions. Nevertheless, these tests are limited because other factors other than bubbles could induce autocorrelation, kurtosis, and skewness. Time-varying risk premiums (Fama and French, 1988) and fads (Poterba and Summers, 1988) could also cause autocorrelation. Finally, asymmetric fundamental news could induce skewness while the flow of information into the market can create leptokurtosis (Tauchen and Pitts, 1983).

For the second category, researchers use the explosiveness test to detect a bubble's presence (Chan et al., 1998). This test examines a particular bubble footprint - explosiveness of returns that cause local price peaks of suspected bubble episodes. This analysis becomes anecdotal because this test only uses portions of the data set. Consequently, researchers should use this test in conjunction with other tests.

\footnotetext{
${ }^{4}$ Daily returns would be very noisy making it very difficult to detect bubbles. In fact McQueen and Thorley (1994) suggest the use of monthly rather than weekly returns to test duration dependence since weekly returns contain more noise than the former.

${ }^{5}$ Hatipoglu and Uyar (2012) suggest that the bubbles might spill over from the developed markets to the emerging markets.
} 
For the third category, researchers have applied cointegration analysis and regime-switching tests to test for price deviation from fundamentals. In theory, the fundamental variable such as stock prices, dividends, and earnings would be an I(1) process and thus be cointegrated. Consequently, stock prices would grow as a multiple of dividends or earnings but a growing bubble would obscure the long-term relationships between stock prices, dividends, and earnings. Cointegration analysis could however, have trouble detecting bubbles because it is effectively a joint test of correct model specification and the existence of a bubble (Brooks and Katsaris, 2003). Moreover, if a bubble periodically collapses, then the cointegration tests could fail to detect it (Evans, 1991). Finally, the lack of cointegration is not a sufficient condition for proving the existence of bubbles because other factors could weaken the cointegration between stock prices and fundamental variables such as large and persistent shocks (Johansen, 1991) and changes in economic regimes (Chow, 1998). Finally, the cointegration test possesses little power if researchers use short time spans (Brooks and Katsaris, 2003, Pierse and Snell, 1995).

Another test of price deviation from fundamentals involves the use of the regime-switching test of Hamilton (1989) as applied in Engel and Hamilton (1989) and Van Norden and Schaller (1993) to examine trends in time series and switches in trends. Ahmed, Li and Rosser (2006) and Ahmed et al. (2010) use this methodology to test for bubbles by first estimating a vector autoregressive model (VAR) as a measure of the presumptive fundamental. The residuals from the resulting VARs are then used for the bubble test, i.e., test for the absence of movements away from fundamentals. This approach however suffers from the same problem as cointegration tests, that of the misspecified fundamental.

For the last category, McQueen and Thorley (1994) developed the duration dependence test that overcomes the many restrictions of traditional bubble tests. First, the test analyses a unique characteristic of rational bubbles - negative duration dependence, which means the conditional probability of a positive run of returns ending continually falls given the run has made it to the current period. Second, unlike cointegration tests, researchers do not need to specify the relationships in the model, and this model overcomes the problem of non-linearity by allowing the parameters to vary. Finally, the time series being investigated does not need to be normally distributed for the duration dependence test.

\section{Data and methods}

We collect from DataStream the closing prices for the MSCI Singapore Index from January 1970 to March 2013 and MSCI Indonesian Index from January 1988 to March 2013. 
We test for the existence of rational speculative bubbles by using descriptive statistics, explosiveness tests and the duration dependence test. The descriptive statistics include skewness, kurtosis, and autocorrelation. As discussed earlier, we would expect the coefficient of skewness to be negative in the presence of bubbles that eventually burst. Furthermore, the growth of bubble induces leptokurtosis due to the mixing of distributions. A bubble would also induce positive autocorrelation in the returns. We use the Ljung-Box statistics, Q(12), to test the first 12 autocorrelation coefficients jointly. The Jarque-Bera statistic is used additionally to test for the normality of the distributions.

For the explosiveness tests, we follow Chan et al. (1998) and check for bubble footprints. Since we use only a portion of the data, this test becomes anecdotal. We identify the local maxima in the weekly returns. Rising or explosive returns before these local peaks suggest the presence of a rational bubble. We investigate this relationship by estimating the following regression in (1):

$R_{t}=\alpha+\beta T_{t}+\varepsilon_{t}$

where $R_{t}$ equals the monthly or weekly returns while $T_{t}$ represents the number of months or weeks from the bubble's beginning. We selected three starting dates of bubbles, which correspond 18, 24 and 30 months from the beginning of the local maxima.

For the duration dependence test, we first test for rational bubbles using nominal returns. For robustness tests, we follow Chan et al. (1998) and also use excess returns, defined with respect to the sample mean, and $\mathrm{AR}(4)$ residuals. ${ }^{6}$

McQueen and Thorley (1994) proposed using monthly rather than weekly returns for the duration dependence tests because weekly returns contain more noise, which would make detecting bubbles more difficult. However Harman and Zuehlke (2004) report that duration dependence test results are sensitive whether one uses monthly or weekly returns based on U.S. data. Lehkonen (2010) finds the same in China. Hence, we use both monthly and weekly returns in our analysis.

We follow McQueen and Thorley (1994) and convert returns into positive and negative run lengths. For example, if we have returns that comprises of four positive returns followed by three negative, and then five

\footnotetext{
${ }^{6}$ As additional robustness tests we also use real returns, real excess returns, and AR(4) residuals based on real returns with similar results. We do not report them here to save space but are available upon request.
} 
positive and two negative returns. We encode these returns into the data set as positive runs with values of 4 and 5 and negative runs with values of 3 and 2 . The run lengths are new series.

We compute the sample hazard rate for each run length $i$ by calculating $h_{i}=\mathrm{N}_{\mathrm{i}} /\left(\mathrm{M}_{\mathrm{i}}+\mathrm{N}_{\mathrm{i}}\right)$, where $\mathrm{N}_{\mathrm{i}}$ represents the completed number of runs of length $i$ in the sample and $\mathrm{M}_{\mathrm{i}}$ equals the completed number of runs that exceed $i$. This is derived from maximizing the hazard version of the log-likelihood function (2) as defined by McQueen and Thorley (1994) with respect to $h_{i}$. w.

$$
L\left(\theta \mid S_{T}\right)=\sum_{i=1}^{\infty} N_{i} \ln h_{i}+M_{i} \ln \left(1-h_{i}\right)+Q_{i} \ln \left(1-h_{i}\right)
$$

$\mathrm{Q}_{\mathrm{i}}$ equals the partial runs with the length exceeding $i$ and occurs at the beginning or end of the data set, while $\mathrm{T}$ is the number of observations. If a time series contains a rational bubble, then the sample hazard rate $\left(h_{i}\right)$ falls monotonically with the length of run $i$. We formally test this relationship by defining a log-logistic function for the hazard function in (3) (Chan et al., 1998, Yu and Sz, 2003). The log-logistic function bounds the probability space for the $h_{i}$ parameter between $(0,1)$ (Yu and Sz, 2003).

$$
h_{i}=\frac{1}{1+e^{-(\alpha+\beta \ln i)}}
$$

Using a logit regression, we estimate the parameters of the hazard function, where the log of the current run length becomes the independent variable, while the dependent variable is a dummy variable. ${ }^{7}$ The dependent variable equals one if the run ends in the next period and 0 if the run continues.

A non-decreasing hazard rate would imply the time series has no rational bubbles. Failing to reject the null hypothesis, $\mathrm{H}_{0}: \beta=0$, indicates no rational speculative bubble and means that the hazard rate is independent of the length of a run $i$. The alternative hypothesis, $\mathrm{H}_{1}: \beta<0$, suggests negative duration dependence because the probability of a run ending falls with the run's length. Under a true null hypothesis, $\mathrm{H}_{0}: \beta=0$, the likelihood ratio test (LRT) is asymptotically distributed as a chi-square with one degree of freedom:

$L R T=2\left[\log\right.$ unrestricted $-\log$ restricted $\left.\sim \chi_{1}^{2}\right]$

\section{Empirical results}

\footnotetext{
${ }^{7}$ Duration dependence test uses non-linear estimates to test the presence of speculative bubbles. Nonlinear estimation might provide multiple maxima of the likelihood function. Since we use logit regression, it does not provide more than one maxima (see, Altman, Gill and McDonald, 2004).
} 


\subsection{Descriptive statistics}

We show the monthly MSCI Singapore Index in Figure 1 and monthly MSCI Indonesian Index in Figure 2. The Singaporean Index conveys several boom and bust cycles. The index rises until January 1995 and then plummets from the Asian Financial Crisis that begun in Thailand in July 1997. During the crisis, the MSCI Singapore Index plunged 59\% from a high of 1,334 points in February 1996 to a low of 541 points in August 1998. Subsequently, the index again plunged during the global finance crisis in $2007 / 2008$ and started rising again. However, it has not surpassed its peak of October 2007. On the other hand, the Indonesian Index hovered around 600 points and began growing after 2002 until February 2008 when the Global Financial Crisis struck the world. Figure 2 shows the index increasing, which coincides with the U.S. housing bubble. In 2010, the Indonesian index has recovered and surpassed its peak.

Table 1 provides the descriptive statistics of the Singaporean stock market for nominal monthly and weekly returns while Table 2 shows the descriptive statistics for the Indonesian stock market. The Singapore Index averaged a monthly return of $0.859 \%$, and attained a maximum of $47.092 \%$ and a minimum of $-41.950 \%$ while the Indonesian Index averaged a monthly return of $2.002 \%$ with a high of $94.943 \%$ and a low of $34.916 \%$. The average weekly return for Singapore was $0.14 \%$, attaining a maximum of $19.24 \%$ and a minimum of $-34.68 \%$ while Indonesia averaged $0.425 \%$ with a high of 69.537 and a low of $-21.273 \%$.

Both the monthly and weekly returns for Indonesia and the monthly returns for Singapore have positive skewness, but Singapore's weekly return has negative skewness. Positive skewness indicates the lack of a bubble. Both monthly and weekly returns for Singapore and Indonesia have significant, positive kurtosis coefficients that convey leptokurtosis. Leptokurtosis means the returns cluster around the mean with fat tails and suggests the existence of a bubble. From Tables 1 and 2, the significant Jarque-Bera test statistics indicate both monthly and weekly returns for Singapore and Indonesia do not originate from a normal distribution, another indicator of a bubble. For both monthly and weekly returns for Singapore, the autocorrelation coefficients and the Ljung-Box statistics, Q(12), show serial correlation that signals the presence of bubbles. For Indonesia, the Ljung-Box statistic for weekly returns is statistically significant but the monthly returns are not.

Except for the skewness and the Ljung-Box statistic for monthly returns, the rest of the descriptive statistics for Indonesia suggest the presence of bubble footprints, while all descriptive statistics for Singapore, except for the skewness coefficient of monthly returns, also suggest the presence of bubble footprints. This begs the question: Did rational speculative bubbles cause these footprints? 


\subsection{Explosiveness tests}

Before we formally test for the presence of rational bubbles, we search for evidence of explosive growth prior to the local peaks in the Singaporean and Indonesian stock indices. We observe five local peaks in the Singapore Index and marked them accordingly in Figure 1. The local peaks occurred in January 1973, June 1981, February 1996, December 1999, and October 2007. We also observe two local peaks in the Indonesia Index in Figure 2. These occurred in October 2007 and March 2013.

We report the estimated $\beta$ parameters in equation (1) for Singapore in Table 3 and for Indonesia in Table 4. Only positive $\beta$ s convey the existence of rational bubbles. The t-statistics are reported in parenthesis below the $\beta$ parameter estimates, and statistically significant positive $\beta$ s are marked in bold. Thus, we observe a bubble footprint for the Singapore Index for the 24-month weekly returns leading to the December 1999 peak. Similarly, we find a bubble footprint for the Indonesia Index for the 18-month returns leading to the October 2007 peak just before the GFC. However, a visual check of Figures 1 and 2 shows that the crash side of these apparent bubble footprints are not instantaneous as would be expected when a bubble bursts casting doubt on whether or not these were actually bubble episodes. In fact the Singapore index takes approximately 9 months to bottom out while the Indonesian index takes about 12 months. Interestingly, the GFC does not show up in the explosiveness test for Singapore, irrespective whether we used monthly or weekly returns. We use the duration dependence test in the next section to formally test for the presence of rational speculative bubbles.

\subsection{Duration dependence test}

\subsubsection{Monthly returns.}

First we conduct duration dependence tests over the period from 1975:01 to 2007:01 corresponding with Rangel and Pillay's (2007) sample period for Singapore. Rangel and Pillay (2007) report the absence of rational bubbles in positive runs for Singapore over this period. Though Rangel and Pillay (2007) use the Singapore Strait Times Index (STI) calculated by Datastream while we use the MSCI Singapore Index, we report very similar values for the beta coefficient in the log-logistic hazard function (equation 3). We calculated a beta 
coefficient of -0.0759 for positive runs compared with -0.0326 in Rangel and Pillay (2007). ${ }^{8}$ More importantly, both our beta coefficients are not statistically significant indicating the absence of rational bubbles. ${ }^{9}$

Next we attempt to replicate Yu and Hassan's (2009) duration dependence test results for Indonesia over the period 1992:01 to 2003:03. Yu and Hassan (2009) used the S\&P/IFCG price index for Indonesia and semi-parametric Cox and parametric proportional (i.e., Exponential, Weibull, Gompertz) hazard functions and report the absence of rational bubbles. We use the MSCI Indonesia Index and the log-logistic hazard function and report a beta coefficient of 0.1744 that is not statistically significant. ${ }^{10}$ Therefore consistent with $\mathrm{Yu}$ and Hassan (2009) we also do not find evidence of rational bubbles in Indonesia over the period from 1992 to 2003.

Now we conduct the tests for our full sample period. We report the run counts, hazard rates and the duration dependence test for monthly returns in Table 5 for Singapore and in Table 6 for Indonesia. Panel A in Tables 5 and 6 show the results for nominal returns. The second and fourth columns of Panel A in Table 5 show that of the 519 monthly returns over the period from 1970:01 to 2013:03 for Singapore, 128 were followed by a return of the opposite sign (i.e., runs of 1) and 47 were followed by a return of the same sign (i.e., runs of 2). The second and fourth columns of Panel A in Table 6 show that of the 303 monthly returns over the period from 1988:01 to 2013:03 for Indonesia, 62 were followed by a return of the opposite sign (i.e., runs of 1) and 34 were followed by a return of the same sign (i.e., runs of 2). Singapore experienced the longest positive run that lasted 14 months while Indonesia experienced a 10-month positive run. Singapore's bull run started in March 1971 and lasted until April 1972. Assuming independent monthly returns, the probability of experiencing a bull run lasting 14 months, equals $0.006 \%$, or 1 in 16,000, which suggests a bubble footprint in Singapore. In the same way, the probability of experiencing a bull run lasting 10 months, equals $0.097 \%$ or 1 in 1,000 which also suggests a faint bubble footprint in Indonesia.

Testing for rational bubbles, we examined the patterns in the hazard rates shown in the third column in Panel A of Tables 5 and 6 . Hazard rates that continuously fall given a run has made it $i$ periods, indicate rational bubbles. For both Singapore and Indonesia, the hazard rates oscillate upward and downward, which contradicts the existence of rational bubbles. ${ }^{11}$

\footnotetext{
8 The detailed results are not reported here to save space but are available from the authors upon request.

${ }^{9}$ Rangel and Pillay (2007) report a significantly negative beta coefficient of -0.4387 for negative runs. We also calculate a negative beta coefficient of -0.0406 but it is not statistically significantly. The difference in magnitude and significance of our results with theirs could be due to the difference in the indices used.

${ }^{10}$ The detailed results are not reported here to save space but are available from the authors upon request.

${ }^{11}$ Although negative bubbles cannot exist since security prices are bounded, we also report in Tables 5 and 6 , the negative runs for completeness.
} 
We calculated the likelihood ratio test statistic and examined whether the beta coefficients in equation (3) significantly differ from zero. A statistically significant, negative beta coefficient gives evidence of a rational bubble, while an insignificant coefficient conveys its absence. Examining the bottom of Panel A in Table 5 we find the beta coefficient for Singapore, at -0.228 , to have the right sign but it is not statistically significant. In Panel A of Table 6 we find the beta coefficient for Indonesia with not only the wrong sign at 0.09, but is also not statistically significant. Thus, the results from the duration dependence test on monthly nominal returns do not support the presence of rational bubbles in either Singapore or Indonesia.

As robustness tests, we also report in Tables 5 and 6 the results using nominal monthly excess returns in Panel B and the AR(4) residuals in Panel C. For Singapore, the longest positive run of excess returns lasted seven months, while the longest positive run of AR(4) residuals survived 13 months. Indonesia experienced the longest run of six months for excess returns and seven months for AR(4) residuals. Similar to Panel A, the sample hazard rates oscillate upward and downward with no discernible pattern. The formal test on the beta coefficients of the log-logistic hazard functions also support the absence of a bubble. For Singapore, the beta coefficient for excess returns equals 0.06 while the coefficient for $\operatorname{AR}(4)$ residuals is -0.112 , and both do not statistically differ from zero, even though the coefficient for AR(4) has the correct sign. Nevertheless, Indonesia does have a statistically significant beta coefficient for excess returns, but the beta is positive which is not consistent with a rational bubble. The results from Panels B and C of Tables 5 and 6 are consistent with the results of Panel A and do not support the existence of rational speculative bubbles in either Singapore or Indonesia using monthly returns. ${ }^{12}$

We also conducted duration dependence tests on monthly returns over the sub-periods 1988 to 1997 and 1990 to 2006 in order to compare our results with those of Sarno and Taylor (1999) who used cointegration tests over the period from 1988 to 1997 and Ahmed et al. (2010) who employed regime-switching models over the period from 1990 to $2006 .{ }^{13}$ Contrary to their findings, we do not find evidence of rational bubbles in either Singapore or Indonesia. ${ }^{14}$ This indicates that the results of cointegration and regime-switching tests are not necessarily consistent with results of duration dependence tests.

\footnotetext{
${ }^{12} \mathrm{We}$ also performed the same tests on real monthly returns with similar results. These results are available from the authors upon request.

${ }^{13}$ Our results remain robust even when we use a sub-sample from 1988-2007, including December 2007. Furthermore, as a robustness test we divide our sample into subsamples based on each suspected bubble episode, and our results remain robust. We saved space by not reporting the detailed results but these are available from the authors upon request.

${ }^{14}$ The detailed results are not reported here to save space but are available from the authors upon request.
} 


\subsubsection{Weekly returns.}

We now switch our focus to weekly returns to test the sensitivity of our duration dependence test results to data frequency. Table 7 shows the duration dependence tests for Singapore while Table 8 displays the results for Indonesia. The second and fourth columns of Panel A in Table 7 show that of the 1,737 weekly returns for Singapore, 411 were followed by a return of the opposite sign (i.e., runs of 1) and 203 were followed by a return of the same sign (i.e., runs of 2). The second and fourth columns of Panel A in Table 8 show that of the 1,316 weekly returns for Indonesia, 312 were followed by a return of the opposite sign (i.e., runs of 1) and 141 were followed by a return of the same sign (i.e., runs of 2). Furthermore, Panel A of Table 7 shows that Singapore had the longest positive run lasting 10 weeks while Panel A of Table 8 shows the bull run for Indonesia lasted 15 weeks. Again, if we assume independent weekly returns, the probability of a run lasting 10 (15) weeks is $0.1 \%(0.003 \%)$ or about 1 in $1000(33,333)$, which suggest the presence of bubble footprints in both Singapore and Indonesia.

Examining the beta coefficients at the bottom of Panel A of Table 7, we find the beta for Singapore at 0.103 exhibiting the correct sign, but it is not statistically significant. This indicates the absence of rational bubbles. However, the beta coefficient for Indonesia at -0.230 reported in Panel A of Table 8 not only exhibits the correct sign, but is also statistically significant. This signifies the presence of rational bubbles in Indonesia. ${ }^{15}$ Our results suggest that the rational bubble episode in Indonesia would have most likely occurred during the GFC as suggested by the explosiveness test result reported earlier in Table 4.

As robustness tests, we report in Panels B and C in Tables 7 and 8 the results for nominal weekly excess returns and AR(4) residuals, respectively. Singapore experienced a bull run lasting nine weeks for both nominal excess returns and AR(4) residuals while Indonesia witnessed a bull run of 11 weeks for nominal excess returns and 12 weeks for AR(4) residuals. Similar to Panel A, Singapore has beta coefficients of -0.056 for nominal excess returns and -0.001 for $\mathrm{AR}(4)$ residuals. Though they have the correct sign, the coefficients are not statistically significant. On the other hand, Indonesia has significantly negative beta coefficients of 0.276 for nominal excess returns and -0.291 for $\mathrm{AR}(4)$ residuals. These results support those reported in Panel

\footnotetext{
15 We avoid discussing the findings for negative runs because security prices are bounded and cannot experience negative bubbles. However, we report the negative run results for completeness.
} 
A. Therefore using weekly returns, we find evidence of rational speculative bubbles in Indonesia but not for Singapore. ${ }^{16}$

As we did with monthly returns, we also conducted duration dependence tests on weekly returns over the sub-periods 1988 to 1997 and 1990 to 2006 to compare our results with Sarno and Taylor (1999) and Ahmed et al. (2010). Over these sub-periods we do not find evidence of rational bubbles in either Singapore or Indonesia again contradicting the findings of Sarno and Taylor (1999) and Ahmed et al. (2010) which were obtained using cointegration and regime-switching tests, respectively. ${ }^{17}$ In view of the shortcomings of the cointegration and regime-switching tests discussed earlier, we suggest that no rational bubbles existed in either Singapore or Indonesia over the period 1988 to 2006.

In sum, using duration dependence tests our results showed no signs of rational speculative bubbles in the Singaporean stock market over the period from 1970 to 2013, whether using monthly or weekly returns. In contrast, our results for Indonesia showed no indication of rational speculative bubbles over 1988 to 2013 when we use monthly returns, but we find a strong presence of rational speculative bubbles when we use weekly returns. This highlights the sensitivity of the duration dependence tests to data frequency consistent with the findings of Harman and Zuehlke (2004) and Lehkonen (2010). We also find that the results of cointegration and regimeswitching tests for rational bubbles conducted earlier by Sarno and Taylor (1999) and Ahmed et al. (2010) are not fully consistent with the results from duration dependence tests.

However, a major limitation of our study, indeed that of the duration dependence test of McQueen and Thorley (1994) is that it based on only one rational bubble model, that of Blanchard and Watson (1982), though one that is the most commonly used in the literature. We also used only one specification of the hazard function, i.e., the log-logistic specification. In addition, we acknowledge that there are recent advances in other bubble tests as in Taylor and Peel (1998) and Enders and Siklos (2001) and so we do not claim our results to be comprehensive. $^{18}$

\section{Concluding remarks}

\footnotetext{
${ }^{16} \mathrm{We}$ also performed the same tests on real weekly returns with similar results. These results are available from the authors upon request.

17 The detailed results are not reported here to save space but are available from the authors upon request.

${ }^{18}$ See Bohl (2003) and Payne and Waters (2007) for recent applications of these techniques.
} 
The media have suggested the existence of asset bubbles in Asian stock markets because these markets experienced several episodes of plummeting prices after a spurt of rapid price growth. Since previous studies offered contradictory evidence on the Singaporean and Indonesian stock markets, we re-examined the data and searched for rational speculative bubbles using an expanded data set that contains at least two possible bubbles The Asian Financial Crisis (AFC) of 1997 and the Global Financial Crisis (GFC) of 2007-2008. By using descriptive statistics, explosiveness test and the duration dependence test, we searched for the existence of rational speculative bubbles over the period 1970-2013 for Singapore and 1988-2013 for Indonesia. Inasmuch as it is claimed that the duration dependence test is theoretically superior to other available tests for bubbles we wanted to test its efficacy in practice. The inclusion of the at least another suspected bubble episode in our sample period adds power to our duration dependence tests relative to earlier studies conducted by Rangel and Pillay (2007) and Yu and Hassan (2009). Our duration dependence test results rule out the presence of rational bubbles in Singapore during our sample period in either weekly or monthly returns. However, for Indonesia our duration dependence test results rule out the presence of bubbles in monthly but not in weekly data. Thus the result from the Indonesian stock market illustrates the sensitivity of duration dependence test results on data frequency.

We contribute to the literature by extending the results of Rangel and Pillay (2007) for Singapore. We extend their results using an expanded sample period that contains at least one more additional suspected bubble episode, effectively increasing the power of our duration dependence test, as well as using both weekly and monthly returns. We also extend the results of Yu and Hassan (2009) and present new evidence, with weekly data, of the presence of bubble episodes in Indonesia in our extended sample period.

Finally, we also contribute to the literature on bubble testing by presenting new evidence supporting the earlier claim by Harman and Zuehlke (2004) and Lehkonen (2010) that though the duration dependence test appears to be theoretically superior to the other bubble tests, its results could be sensitive to data frequency suggesting that the duration dependence test results are not always conclusive and that it should be used in conjunction with other tests. 


\section{References}

Ahmed, E., H. Li and J. B. Rosser (2006), 'Nonlinear bubbles in Chinese stock markets in the 1990s', Eastern economic journal, 1-18.

Ahmed, E., J. B. Rosser and J. Y. Uppal (2010), 'Emerging markets and stock market bubbles: Nonlinear speculation?', Emerging Markets Finance and Trade, 46, 23-40.

Altman, M., J. Gill and M. P. Mcdonald (2004), Numerical issues in statistical computing for the social scientist: John Wiley \& Sons.

Blanchard, O. J. and M. W. Watson (1982), Bubbles, rational expectations and financial markets. National Bureau of economic research Cambridge, Mass., USA.

Bohl, M. T. (2003), 'Periodically collapsing bubbles in the US stock market?', International Review of Economics \& Finance, 12, 385-97.

Brooks, C. and A. Katsaris (2003), 'Rational speculative bubbles: an empirical investigation of the London Stock Exchange', Bulletin of Economic Research, 55, 319-46.

Chan, K., G. Mcqueen and S. Thorley (1998), 'Are there rational speculative bubbles in Asian stock markets?', Pacific-Basin Finance Journal, 6, 125-51.

Chow, Y. F. (1998), 'Regime switching and cointegration tests of the efficiency of futures markets', Journal of Futures Markets, 18, 871-901.

Enders, W. and P. L. Siklos (2001), 'Cointegration and threshold adjustment', Journal of Business \& Economic Statistics, 19, 166-76.

Engel, C. and J. D. Hamilton (1989), Long swings in the exchange rate: Are they in the data and do markets know it? : National Bureau of Economic Research.

Evans, G. W. (1991), 'Pitfalls in testing for explosive bubbles in asset prices', The American Economic Review, $922-30$

Fama, E. F. and K. R. French (1988), 'Permanent and temporary components of stock prices', The Journal of Political Economy, 246-73.

Hamilton, J. D. (1989), 'A new approach to the economic analysis of nonstationary time series and the business cycle', Econometrica: Journal of the Econometric Society, 357-84.

Harman, Y. S. and T. W. Zuehlke (2004), 'Duration dependence testing for speculative bubbles', Journal of Economics and Finance, 28, 147-54. 
Hatipoglu, O. and O. Uyar (2012), 'Do bubbles spill over? Estimating financial bubbles in emerging markets', Emerging Markets Finance and Trade, 48, 64-75.

Huddart, S. and H. Louis (2007), 'Stock returns, earnings management, and insider selling during the 1990s stock market bubble', Unpublished working paper. Pennsylvania State University.

Johansen, S. (1991), 'Estimation and hypothesis testing of cointegration vectors in Gaussian vector autoregressive models', Econometrica: Journal of the Econometric Society, 1551-80.

Lehkonen, H. (2010), 'Bubbles in china', International Review of Financial Analysis, 19, 113-17.

Mcqueen, G. and S. Thorley (1994), 'Bubbles, stock returns, and duration dependence', Journal of Financial and Quantitative Analysis, 29, 379-401.

Payne, J. E. and G. A. Waters (2007), 'Have equity REITs experienced periodically collapsing bubbles?', The Journal of Real Estate Finance and Economics, 34, 207-24.

Pierse, R. G. and A. J. Snell (1995), 'Temporal aggregation and the power of tests for a unit root', Journal of Econometrics, 65, 333-45.

Poterba, J. M. and L. H. Summers (1988), 'Mean reversion in stock prices: Evidence and implications', Journal of financial economics, 22, 27-59.

Ramiah, V. and M. Graham (2013), 'The impact of domestic and international terrorism on equity markets: evidence from Indonesia', International Journal of Accounting \& Information Management, 21, 91-107.

Rangel, G. and S. S. Pillay (2007), Evidence of bubbles in the Singaporean stock market. Singapore Economic Review Conference.

Sarno, L. and M. P. Taylora (1999), 'Moral hazard, asset price bubbles, capital flows, and the East Asian crisis: the first tests', Journal of International Money and finance, 18, 637-57.

Schiller, R. J. (2000), 'Irrational exuberance', Princeton University Press.

Tauchen, G. E. and M. Pitts (1983), 'The price variability-volume relationship on speculative markets', Econometrica: Journal of the Econometric Society, 485-505.

Taylor, M. P. and D. A. Peel (1998), 'Periodically collapsing stock price bubbles: a robust test', Economics Letters, $61,221-28$.

Van Norden, S. and H. Schaller (1993), 'The predictability of stock market regime: evidence from the Toronto Stock Exchange', The Review of Economics and Statistics, 505-10.

Wang, J. (2011), 'Transient institutional investors and insider trading signals', International Journal of Accounting \& Information Management, 19, 118-45. 
Yu, I. and A. Sz (2003), 'Testing for bubbles in the hong kong stock market', Hong Kong Monetary Authority.

Yu, J.-S. and M. K. Hassan (2009), 'Rational speculative bubbles in the OIC (Orgnisation of Islamic Conference) stock markets', International Journal of Economics, Management and Accounting, 7. 